\title{
Design of Light Source and Light Controller Used for Bottle Defect Detection System
}

\author{
Chunhui Liu', ${ }^{1, a}$, Lili Zhang ${ }^{2, b}$, Wenchao Huang ${ }^{1}$, Yang Wang ${ }^{1}$ \\ ${ }^{1}$ College of Electrical Engineering and Automation, Shandong University of Science and \\ Technology, Qingdao, 266590, China \\ ${ }^{2}$ College of Electronic Communication and Physics, Shandong University of Science and \\ Technology, Qingdao, 266590, China \\ axhl79@126.com, bsdkd_lili@126.com
}

Keywords: LED light source, Defect inspection, Machine vision, Light controller

\begin{abstract}
The article introduces a kind of LED light source and light controller lighting for the defect inspection system for the beer bottle. The light source includes three kinds of color, red, blue and green. The light controller powers the LED light source, adjusts the brightness and color of LED light source, it can also realize the purpose that PC controls LED light source remotely. External switch trigger circuit of the light controller can control the LED light source on and off according to the assembly line's speed, it can greatly increase the working life of LED light source.
\end{abstract}

\section{Introduction}

The appearance quality of the beer bottle must conform to the relevant provisions, there can't be cracks on the beer bottle wall, and there can't be any stones greater than $1.5 \mathrm{~mm}$, there also can't be defects that seriously affect the appearance of the bottle [1].The traditional way of beer bottle inspection is manual testing. But in modern high-speed filling line, because of the reasons such as visual fatigue, the traditional way of manual testing cannot guarantee the reliability of inspection results with low efficiency .So the intelligent inspection system based on the machine vision inspection technology is used to replace manual testing. The method of machine vision inspection can greatly enhance the production efficiency and improve the automation of production [2]. The machine vision is that doing all kinds of measurement and judgment with machines instead of human eyes. The image capturing device (such as CCD camera) converts the object which will be tested to image signals, and sends it to the special image processing software for identification, inspection and judgment. Then the machine controls the equipment's action according to the result of judgment [3].

As is shown in figure 1, the whole defect inspection system for the beer bottle is mainly includes the following four parts: LED light source, light controller, the image capturing device and the PC (principle computer, including image processing software and source controlling software). The reliability and efficiency of the defect inspection system is largely restricted by the quality of the LED light source and lighting solution. This article introduces a kind of LED light source and light controller lighting for the defect inspection system for the beer bottle based on machine vision technology.

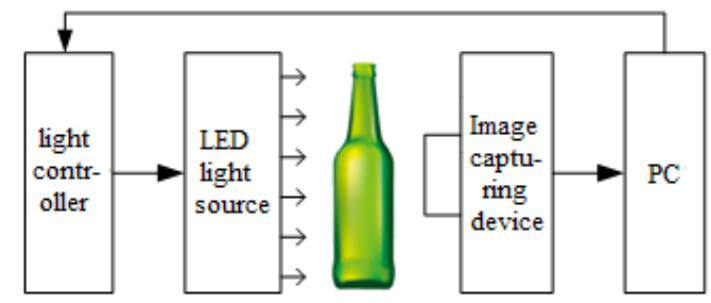

Fig.1: Framework of the defect inspection system 


\section{The design of lighting source}

Lighting environment is one of the important factors which affect the input signal of the machine vision system. It directly affects the quality of the images captured and the processing results of the system [4]. Because there are no universal lighting devices for machine vision, corresponding lighting device must be selected for each specific application examples in order to achieve the best effect [5]. The three-color LED flat light source with red, green and blue has been used for the inspection of beer bottle wall. The product picture of LED lighting source is shown in figure 2.The light distribution outputted by the lighting source is uniform and its brightness and color can both be adjusted according to the requirements of production field. Thus it can insure the best imaging quality and the striking feature.

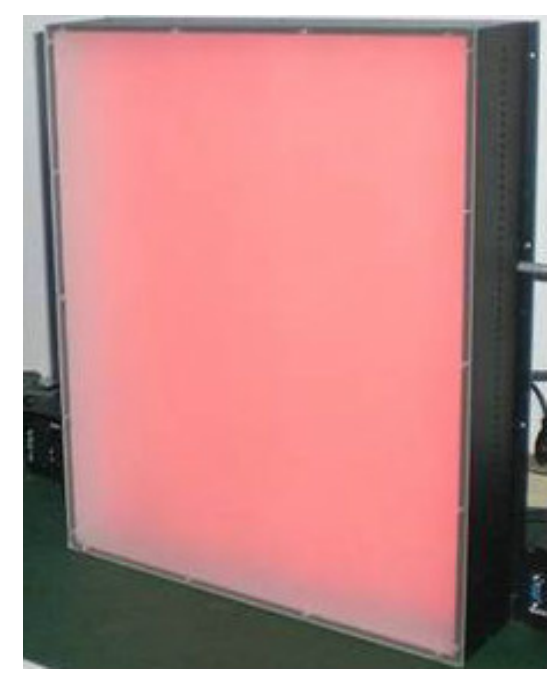

Fig.2: LED lighting source (brightness and color can be adjusted)

\section{The hardware design of light controller}

Light controller is mainly composed of five parts: the MCU and communication circuit, D/A conversion circuit, LED light source driving circuit, the external switch trigger circuit and the power circuit [6]. The structure diagram is shown in the figure 3.

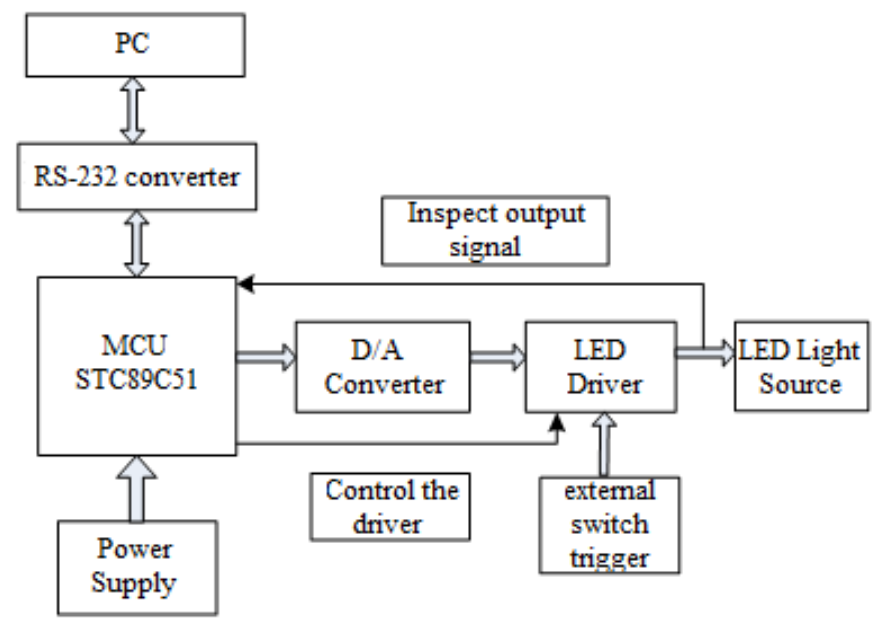

Fig.3: Framework of light controller

When the MCU accepted instructions sent from the PC, it will send data commands to D/A converter. When the D/A converter received the commands, it will select output channels and adjust the output voltage size according to the data commands. The voltage signal will be adjusted by the power amplifying circuit which is composed of LM723C and other electronic components then the amplified voltage signal drives LED light source, so as to change the brightness and color.

The MCU and communication circuit. The MCU and communication circuit is shown in figure 4. As the control chip of the circuit, the STC89C51 MCU's main function is to communicate 
with the computer, adjust the brightness of LED light source, control the LED driver chip's working status, and test LED driving circuit working normally or not.

The RS-232Ccommunication interface of PC works at the voltage of $-15 \mathrm{~V}$ to $+15 \mathrm{~V}$; however, the working voltage of $\mathrm{MCU}$ is $0 \mathrm{~V}$ to $3 \mathrm{~V}$, so a converting circuit between the PC and MCU is required. In this design the chip MAX232 directs the bidirectional signal conversion while they communicate. Chip MAX232 is one of the most common components in communication circuit design, the working voltage of MAX232 is $+5 \mathrm{~V}$. It has two separate RS-232 drivers, and has the stable performance of low power and reliable work.

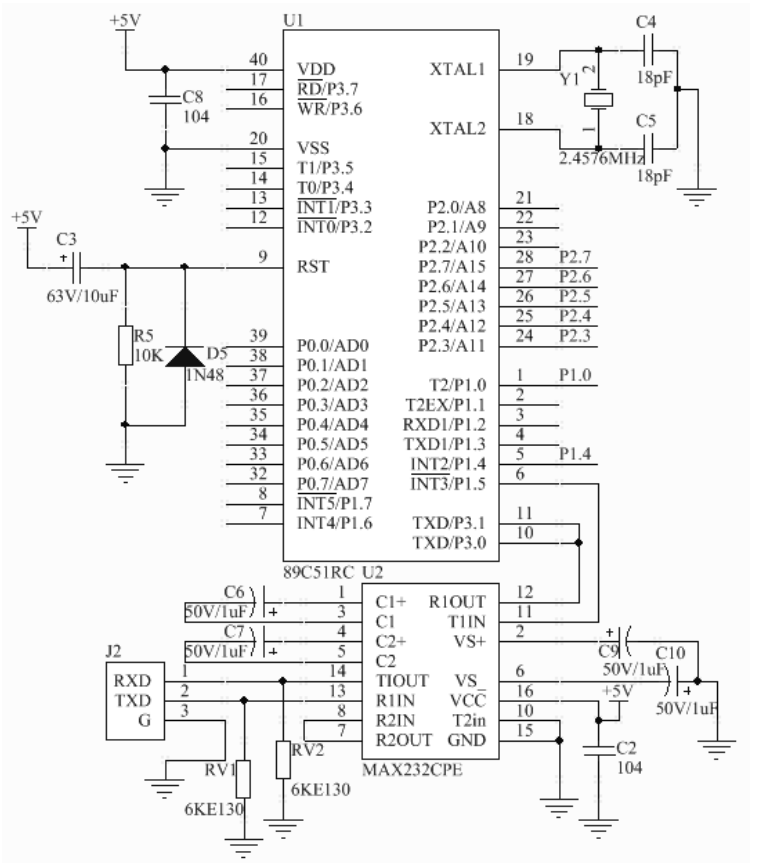

Fig.4: The MCU and communication circuit

D/A converting circuit and LED driving circuit. The data commands sent from PC can be converted to voltage signals by the digital-to-analog converting circuit. As is shown in figure 4, AD7399 is a digital-to-analog converter of 10-bit resolution and four quad serial inputs [7].LM324 is a chip of four separate integrated amplifying circuits, in this circuit it is used as a voltage follower to supply reference voltage to AD7399. The digital-to-analog converter's output voltage calculation formula is shown in formula 1.

$$
V_{\text {out }}=V_{R E F} \times \frac{D_{n}}{2^{10}}
$$

Because the output voltage signal of AD7399 can't directly drive the LED light source, so it is necessary to amplify the signal by the power amplifying circuit which was composed of LM723C and other electronic components. LM723C can output high current while connected with the transistor BU406, only in this way can drive the LED light source [8]. Additionally, the VREF pin of LM723C connected with a variable resistance, the input voltage of LM324 can be regulated by adjusting the variable resistance, making the reference voltage of AD7399 chip changed, so as to regulate the brightness of the LED light source.

AD7399 has four quad serial input, it can output 4 voltage signals, to control four LED drive circuits. It can change the LED color by selecting different channels. The LED drive circuit in figure 5 is only one of the four channels. 


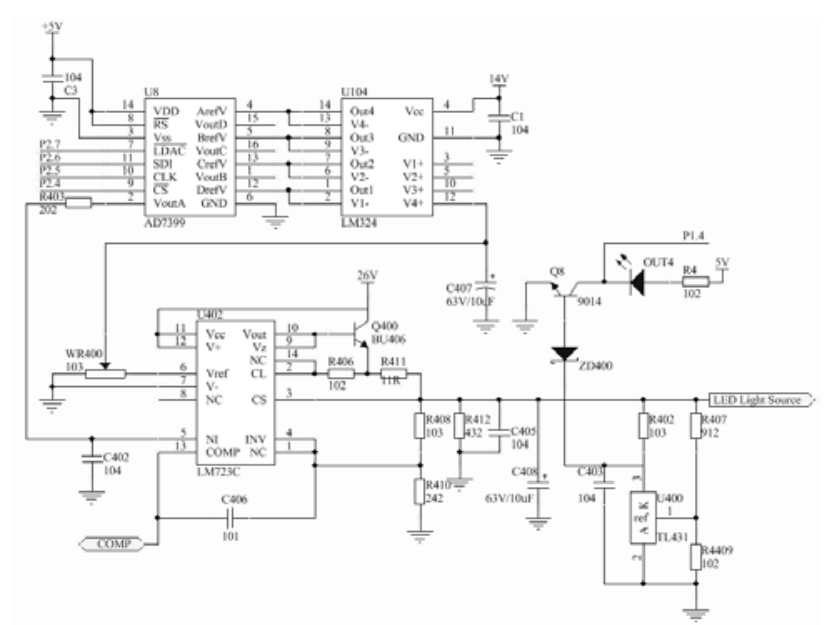

Fig.5: D/A converting circuit and LED driving circuit

External switch trigger circuit. Considering the flow-shop working mode of beer bottle inspection, it is necessary to install a trigger device of external switch in the design. While a bottle passes the device, the switch will be triggered. Then the LED driving circuit will be in working status, LED lights will be turned on.

As is shown in the external switch trigger circuit in figure 6, LM339 is a component integrated four separate voltage comparators, the output state of each comparator changes only if the voltage difference between the two input terminals is greater than $10 \mathrm{mV}$. While the trigger switch TR1 closed, the voltage of positive input terminal is higher than the negative input terminal, the output signal is open-circuit, and the LED driving circuit works normally. While the trigger switch TR1 opened, the voltage of positive input terminal is lower than the negative input terminal, the output signal is low level, the LED driving circuit stops working.

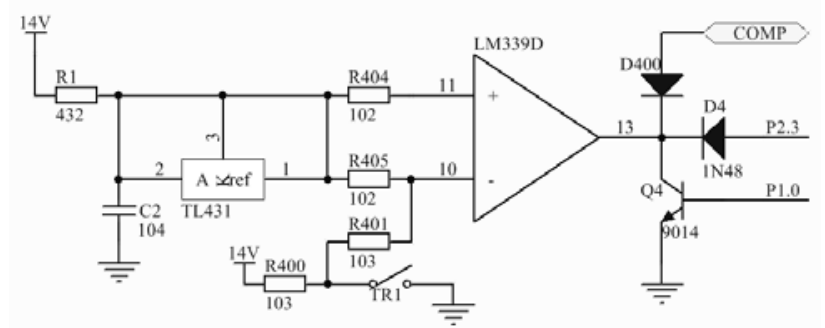

Fig.6: External switch trigger circuit

Power circuit. The main function of power circuit is to provide working voltage to the whole circuit and supply power to LED light source. As is shown in the power supply circuit figure 7, light controller uses a voltage of 26 volts switch power supply.LM317T is a kind of adjustable linear regulator, its output voltage calculation formula is shown in formula 2.

$$
V_{\text {OUT }}=1.25\left(1+\frac{R_{2}}{R_{3}}\right)+I_{2} \times R_{3}
$$

In this circuit, the resistance of R2 is $10 \mathrm{~K}$, the resistance of $\mathrm{R} 3$ is $1 \mathrm{~K}$, and the current of $\mathrm{I} 2$ can be ignored, so the output voltage of LM317T is about $14 \mathrm{~V}$.

Three-terminal integrated regulator $\mathrm{L} 7805 \mathrm{CV}$ is used to generate a voltage of 5 volts, its maximum output current is $1.5 \mathrm{~A}$, and it does not need external compensating elements, and it has over-current protection and thermal-shutdown circuits. 


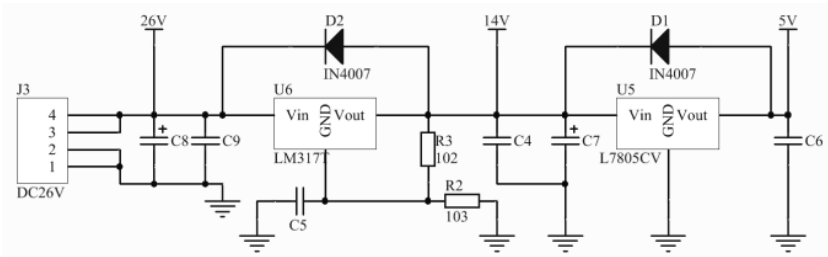

Fig.7: Power circuit

\section{MCU programming}

The MCU of light controller is used to complete the communication with the PC, to control the LED light's trigger mode and adjust its brightness and color .The main program flow chart is shown in figure 8.

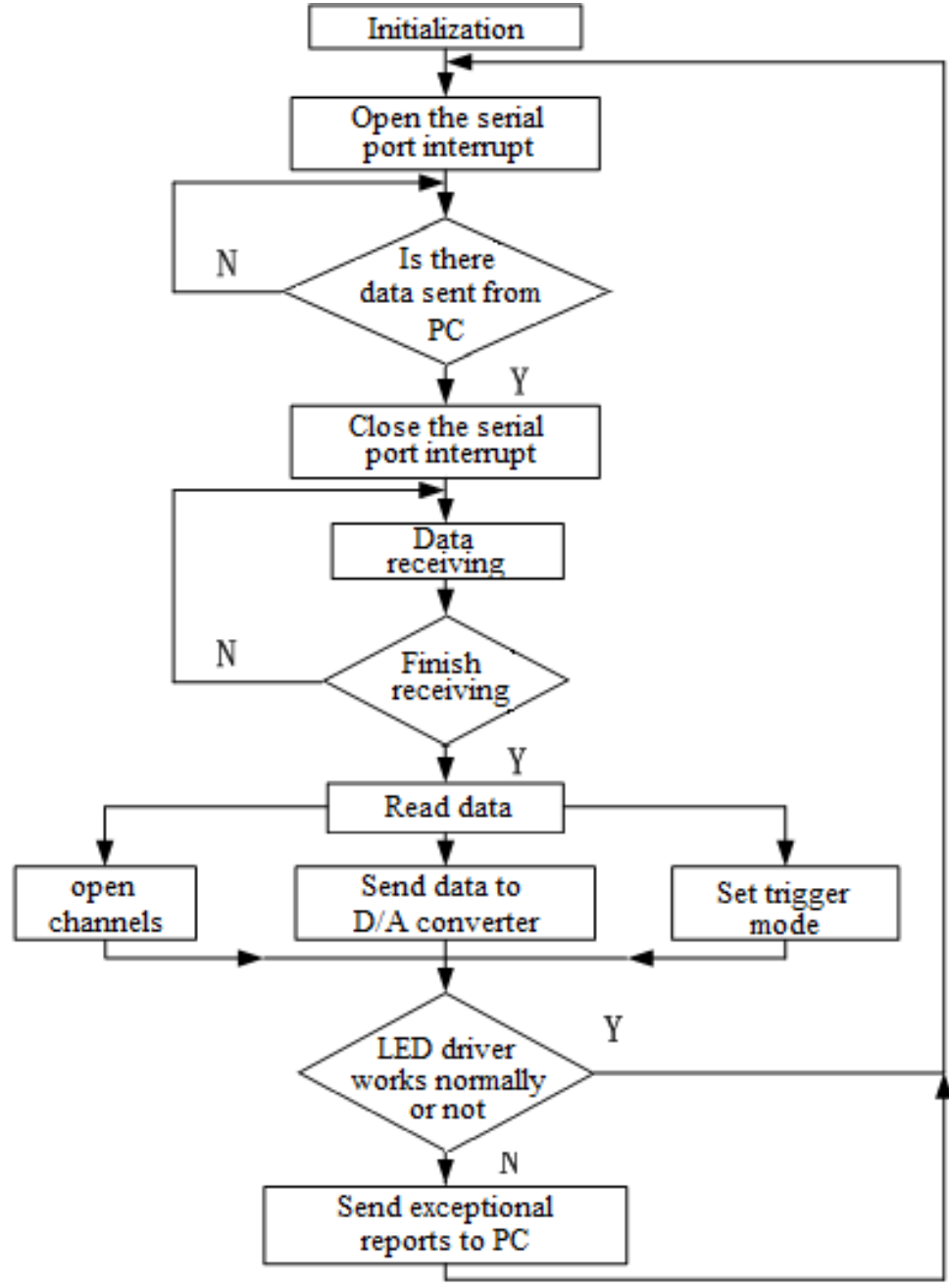

Fig.8: Program flow chart

\section{Conclusions}

The LED light source and light controller introduced in this paper can not only be used in beer bottle defect inspection system, but also be applied in other systems which need LED lighting. LED light source is both energy-saving and environmental protect, it is beautiful, colorful and has high brightness. Light controller uses chip AD7399 of 10-bit resolution, and it can realize high-precision regulation to LED light source. Four channels' output can satisfy different needs for color lighting, it can make sure that the machine vision system captures high-quality images, so as to ensure the reliability of test results. 


\section{Acknowledgements}

The research work was supported by Project of Shandong Province Higher Educational Science and Technology Program (2015).

\section{References}

[1] GB 4544-1996, beer bottles [S].

[2] Zhang Wu-yi, Zhao Qiang-song, Wang Dong-yun. Actualities and developing trend of Machine Vision [J]. Journal of Zhongyuan University of Technology, 12 (1) pp: 9-15, 2008.

[3] Zhang Guangjun. Machine vision [M]. Beijing: Science Press: pp: 3-15, 2005.

[4] LiJun. Study on key technique of light source in machine vision [D]. Tianjin: Tianjin University of Technology, pp: 9-21, 2007.

[5] Wu Wenwei. The study of the opening control system for 6-DOF Robot [D].Changsha: Hunan University,2007.

[6] Geng Yuxin. Hardware circuit design of high precise laser source in fiber-optic communication [D] .Taiyuan: Taiyuan University of Technology, 2010.

[7] Sun Hongxia, Li Tian. Quad serial input 12 bit/ 10 bit DACs AD7398/ AD7399 [J]. International Electronic Elements, 2002.

[8] Ju Dapeng. Study and design of high power LED driver [D]. Suzhou: Soochow University, pp:6- 22,2010. 